TRANSACTIONS OF THE

AMERICAN MATHEMATICAL SOCIETY

Volume 349, Number 9, September 1997, Pages 3569-3584

S 0002-9947(97)01505-5

\title{
SPHERICAL FUNCTIONS ON SYMMETRIC CONES
}

\author{
P. SAWYER
}

\begin{abstract}
In this note, we obtain a recursive formula for the spherical functions associated with the symmetric cone of a formally real Jordan algebra. We use this formula as an inspiration for a similar recursive formula involving the Jack polynomials.
\end{abstract}

\section{INTRODUCTION}

In 1955, Carl S. Herz discussed the hypergeometric functions on the space of real symmetric matrices in [8]. Such functions have been used in multivariate statistical analysis in [11]. More recently, Gross and Richard [4] investigated the subject in a context including complex and quaternionic matrices.

Jordan algebras provide a more general setting for these concepts (see [2], [3] and [17]). Spherical functions, which can be thought as the building blocks of the theory of special functions on these objects, can be defined in this context (see Definition 3.1). Hypergeometric functions can then be expressed in terms of the spherical functions (see [4, page 784]).

If we look at symmetric cones as symmetric spaces, our interest in spherical functions is also rather natural. Indeed the spherical functions play there the role the functions $e^{i u \cdot x}$ play for $\mathbf{R}^{n}$.

Let $V$ be a finite dimension real vector space equipped with a positive definite bilinear form $(\cdot \mid \cdot)$. A cone $\Omega$ in $V$ is proper if $\bar{\Omega} \cap-\bar{\Omega}=\{0\}$, and it is homogeneous if the group of nonsingular linear transformations that fix $\Omega$ is transitive. The dual of a cone $\Omega$ is $\Omega^{*}=\{y \in V:(x \mid y)>0, \forall x \in \bar{\Omega}-\{0\}\}$. A symmetric cone is then an open proper homogeneous cone $\Omega$ such that $\Omega^{*}=\Omega$. If $V$ contains a symmetric cone, a product can be defined such that

(1) $x y=y x$,

(2) $x\left(x^{2} y\right)=x^{2}(x y)$.

With these properties, $V$ is a real Jordan algebra. In addition, it can be shown that

(3) $x^{2}+y^{2}=0$ implies $x=y=0$.

With properties (1), (2) and (3), $V$ is said to be a formally real Jordan algebra.

The symmetric cone then corresponds to the interior of the set of squares of $V$, its "domain of positivity". The process can be reversed. The interior of the set of squares of a formally real Jordan algebra $V$ is a symmetric cone. The space of

Received by the editors March 15, 1994 and, in revised form, April 3, 1995.

1991 Mathematics Subject Classification. Primary 33C55; Secondary 53C35, 17C20, 17C27, $33 \mathrm{C} 45$.

Research supported by a grant from the National Sciences Research Council of Canada.

(C)1997 American Mathematical Society 
positive definite real matrices is a useful example of a symmetric cone to keep in mind.

The rank of $V$ is defined as $r=\max \operatorname{dim}\{\mathbf{R}[x]: x \in V\}$. There are unique polynomials $a_{1}, \ldots, a_{r}$ on $V$ such that $x^{r}-a_{1}(x) x^{r-1}+\cdots+(-1)^{r} a_{r}(x)=0$ for all $x \in V$. We write $\operatorname{tr} x=a_{1}(x)$ and $\operatorname{det} x=a_{r}(x)$.

It can be shown, see for instance [1], that if $V$ is a formally real Jordan algebra, then it has an identity element $e$, and that $(x \mid y)=\operatorname{tr} x y$ is positive definite.

In this paper, we express the spherical functions associated with the symmetric cone of a formally real simple Jordan algebra of rank $r$ in terms of the spherical functions of a Jordan algebra of rank $r-1$. Lemma 3.2 is our first step. The advantage of the formula given there is that it is "coordinate free". In later results, we have to choose a complete system of orthogonal primitive idempotents as explained in Section 2. This is the price we have to pay in order to be able to give the more explicit formula of Theorem 5.3, our first main result.

The work leading to the corresponding result for the positive definite matrices can be found in our unpublished doctoral dissertation. However, the language of Jordan algebras is not used there. With that proviso, the results leading to Theorem 5.3 are new. Our approach is greatly inspired by our interest in symmetric spaces (see Theorem 4.1 and the lemmas leading to its proof).

Theorem 5.3 leads us to the second part of the paper, where we discuss Jack polynomials. These polynomials can be constructed by applying the Gram-Schmidt process to the basis formed by the power-sum symmetric functions with respect to a suitable scalar product which depends on a parameter $\alpha$. We will not expound on this definition, as we will use instead the characterization given at the end of Section 2. There is a well known connection between the spherical functions of symmetric cones and the Jack symmetric polynomials corresponding to certain values of the parameter $\alpha$. Since this parameter is clearly apparent in the formula contained in Theorem $5.3(\alpha=2 / m)$, it is natural to ask whether this formula is valid for other values of the parameter even if the correspondence with symmetric cones is no longer valid. That lead us to the second main result of this paper, Theorem 5.6, which gives an affirmative answer to that question.

A natural question for any result is whether it is any help to the existing theory. We tested our formula against a conjecture of Macdonald's (Conjecture 5.9). This conjecture states that the coefficients of the Jack polynomials when expressed in terms of the basis of elementary symmetric polynomials are themselves polynomial functions of the parameter $\alpha$. This conjecture, if it is true, implies in particular that Jack polynomials can be defined for any value of the parameter. We conclude the paper by obtaining some partial results that are sufficient to settle the case when there are at most three variables (the case of three variables is new).

\section{Some nOtation}

In this paper, the notation referring to Jordan algebras and spherical functions is based on that of Faraut in [2] and in [3]. We will assume that the finite dimensional Jordan algebra $V$ over the real numbers is formally real and simple. For $x, y \in V$, let $(x \mid y)=\operatorname{tr} x y(\operatorname{tr}=\operatorname{trace})$ and let $\|\cdot\|$ be the associated norm. If $c \in V$ is an idempotent then $V(c, \alpha)=\{x \in V: c x=\alpha x\}$. The set of primitive idempotents is denoted by $I_{1}$. Suppose the rank of $V$ is $r$, and that a complete system of orthogonal primitive idempotents $\left\{e_{1}, \ldots, e_{r}\right\}$ is chosen. We then write $V_{i j}=V\left(e_{i}, 1 / 2\right) \cap$ 
$V\left(e_{j}, 1 / 2\right)$ for $i \neq j$ and $m=\operatorname{dim} V_{i j}$ ( $m$ is independent of $i$ and $j$ ). If $x \in V$, its Peirce decomposition with respect to $\left\{e_{1}, \ldots, e_{r}\right\}$ is $x=\sum_{i=1}^{r} \xi_{i} e_{i}+\sum_{i<j} x_{i j}$, where $\xi_{i} \in \mathbf{R}$ and $x_{i j} \in V_{i j}$. The measure on compact spaces will be assumed to give them a total mass equal to 1 .

For the notation referring to partitions and symmetric functions one can refer to [9]. A decreasing sequence of nonnegative integers $s=\left(s_{1}, s_{2}, \ldots\right)$ with only finitely many nonzero terms is said to be a partition of $|s|=\sum s_{i}$. The conjugate of $s$ is $s^{\prime}=\left(s_{1}^{\prime}, s_{2}^{\prime}, \ldots\right)$ where $s_{i}^{\prime}=\operatorname{card}\left\{j: s_{j} \geq i\right\}$. The length of $s$ is $l(s)=$ $\max \left\{i: s_{i} \neq 0\right\}=s_{1}^{\prime}$. Given two partitions $s$ and $t$, we write $s \leq t$ if $|s|=|t|$ and $\sum_{i=1}^{k} s_{i} \leq \sum_{i=1}^{k} t_{i}$ for each $k$. If $l(s) \leq r$, one often writes $s=\left(s_{1}, \ldots, s_{r}\right)$. The partition $(1, \ldots, 1)$ of length $r$ will be denoted by $1^{r}$.

The monomial symmetric function indexed by the partition $s$ can be seen as a function on an arbitrary number of variables such that all but a finite number are equal to 0: if $\lambda_{i}=0$ for $i>r \geq l(s)$ then $m_{s}=m_{s}\left(\lambda_{1}, \ldots, \lambda_{r}\right)=\sum \lambda_{1}^{\sigma_{1}} \cdots \lambda_{r}^{\sigma_{r}}$, where the sum is over all the distinct permutations $\left\{\sigma_{1}, \ldots, \sigma_{r}\right\}$ of $\left\{s_{1}, \ldots, s_{r}\right\}$ and if $l(s)>r$ then $m_{s}\left(\lambda_{1}, \ldots, \lambda_{r}\right)=0$. A symmetric function $f$ is a linear combination of monomial symmetric functions. If $f$ is a symmetric function then $f\left(\lambda_{1}, \ldots, \lambda_{r}, 0\right)=f\left(\lambda_{1}, \ldots, \lambda_{r}\right)$. For each $r \geq 1, f\left(\lambda_{1}, \ldots, \lambda_{r}\right)$ is a symmetric polynomial in $r$ variables. Note that if $N \leq r$, the restriction to $r$ variables is an isomorphism of the symmetric functions of degree at most $N$ and the symmetric polynomials in $r$ variables of degree at most $N$ (see [9, page 12]).

The elementary symmetric function indexed by the partition $s$ is defined by

$$
e_{s}\left(\lambda_{1}, \ldots, \lambda_{r}\right)=\prod_{i=1}^{l(s)} e_{s_{i}}\left(\lambda_{1}, \ldots, \lambda_{r}\right),
$$

where $e_{k}\left(\lambda_{1}, \ldots, \lambda_{r}\right)=\sum_{i_{1}<i_{2}<\cdots<i_{k}} \lambda_{i_{1}} \cdots \lambda_{i_{k}}$ if $r \geq l(s)$ and 0 if $r<l(s)$. The power-sum elementary symmetric function indexed by the partition $s$ is defined by $p_{s}\left(\lambda_{1}, \ldots, \lambda_{r}\right)=\prod_{i=1}^{l(s)} p_{s_{i}}\left(\lambda_{1}, \ldots, \lambda_{r}\right)$, where $p_{k}\left(\lambda_{1}, \ldots, \lambda_{r}\right)=\sum_{i=1}^{r} \lambda_{i}^{k}$. Given a parameter $\alpha$, we can characterize the Jack symmetric function $J_{s}^{(\alpha)}$ by the following three conditions:

$$
\begin{aligned}
J_{s}^{(\alpha)}\left(\lambda_{1}, \ldots, \lambda_{r}\right) & =\sum_{t \leq s} v_{s t}(\alpha) m_{t}\left(\lambda_{1}, \ldots, \lambda_{r}\right), \\
J_{s}^{(\alpha)}(1, \ldots, 1) & =\alpha^{|s|} \prod_{i=1}^{r}((r-i+1) / \alpha)_{s_{i}}, \\
D_{2}(2 / \alpha) J_{s}^{(\alpha)}\left(\lambda_{1}, \ldots, \lambda_{r}\right) & =\gamma(\alpha, r, s) J_{s}^{(\alpha)}\left(\lambda_{1}, \ldots, \lambda_{r}\right)
\end{aligned}
$$

where $(\beta)_{n}=\prod_{i=1}^{n}(\beta+i-1), \gamma(\alpha, r, s)=\sum_{i=1}^{r} s_{i}\left(s_{i}-1+2(r-i) / \alpha\right)$ and

$$
D_{2}(2 / \alpha)=\sum_{i=1}^{r} \lambda_{i}^{2} \frac{\partial^{2}}{\partial \lambda_{i}^{2}}+(2 / \alpha) \sum_{i=1}^{r} \lambda_{i}^{2} \sum_{j \neq i} \frac{1}{\lambda_{i}-\lambda_{j}} \frac{\partial}{\partial \lambda_{i}}
$$

See the remark at the end of $[10, \S 2]$, and $[15$, Theorem 3.1 and 5.4]. Note that these conditions can easily be stated in a way to admit the case $\alpha=0$. We then have $J_{s}^{(0)}=\left(\prod_{i=1}^{r}(r-i+1)^{s_{i}}\right) e_{s^{\prime}}$. Note also that if $r<l(s)$ then $J_{s}^{(\alpha)}\left(\lambda_{1}, \ldots, \lambda_{r}\right)=0$. 


\section{Preliminaries}

Definition 3.1. Fix a complete system of orthogonal primitive idempotents $\left\{e_{1}, \ldots, e_{r}\right\}$ in $V$. For all $x \in V$, there exists $k \in K=(\operatorname{Aut}(V))_{0}$, the connected component of the group of automorphisms of the Jordan algebra $V$ containing the identity, and $\lambda_{j} \in \mathbf{R}, 1 \leq j \leq r$, such that $x=k \sum_{j=1}^{r} \lambda_{j} e_{j}$. Let $V_{j}=$ $V\left(e_{1}+\cdots+e_{j}, 1\right)$ and, for $x \in V$, let $x^{(j)}$ be the orthogonal projection of $x$ on $V_{j}$. Let $\Delta_{j}(x)=\operatorname{det}_{j}\left(x^{(j)}\right)$, where $\operatorname{det}_{j}$ is the determinant relative to the Jordan algebra $V_{j}$. If $s=\left(s_{1}, s_{2}, \ldots, s_{r}\right)$ then let $\Delta_{s}(x)=\Delta_{1}(x)^{s_{1}-s_{2}} \Delta_{2}(x)^{s_{2}-s_{3}} \ldots \Delta_{r}(x)^{s_{r}}$.

Let $\Omega$ be the symmetric cone associated to $V$ (the interior of the set of squares of $V)$. The spherical function of index $s=\left(s_{1}, s_{2}, \ldots, s_{r}\right)$ evaluated at $x \in \Omega$ is given by

$$
\Phi_{s}(x)=\int_{K} \Delta_{s}(k x) d k .
$$

Observe that $\Phi_{s}$ does not depend on the choice of a complete system of orthogonal primitive idempotents, since $K$ is transitive over such systems. For the same reason, $\Phi_{s}(x)$ depends only on the eigenvalues of $x$. We will alternatively write $\Phi_{s}\left(\lambda_{1}, \ldots, \lambda_{r}\right)$ instead of $\Phi_{s}(x)$.

Lemma 3.2. If everything is as in Definition 3.1, then

$$
\Phi_{s}(x)=(\operatorname{det} x)^{s_{r}} \int_{I_{1}} \Phi_{s-s_{r} \cdot 1^{r}}(P(e-c)(x)) d \nu(c)
$$

where $s-s_{r} \cdot 1^{r}=\left(s_{1}-s_{r}, s_{2}-s_{r}, \ldots, s_{r-1}-s_{r}\right)$ and $P$ is the quadratic representation of $V\left(P(y)=2 L(y)^{2}-L\left(y^{2}\right)\right.$, where $L(y)$ denotes the multiplication by $y$ in $\left.V\right)$. The spherical function $\Phi_{s-s_{r} \cdot 1^{r}}$ is defined on the symmetric cone associated to the Jordan algebra $V(c, 0)$.

Proof. Fix $x \in \Omega$. There exists a complete system of primitive idempotents $\left\{e_{1}, \ldots, e_{r}\right\}$ such that $x=\sum_{i=1}^{r} \lambda_{i} e_{i}$. According to the observations following Definition 3.1 , we can use the system $\left\{e_{1}, \ldots, e_{r}\right\}$ to define $\Phi_{s}(x)$. Let $U=\{k \in K$ : $\left.k e_{r}=e_{r}\right\}$. Now,

$$
\begin{aligned}
\int_{K} \Delta_{s}(k x) d k & =\int_{K} \Delta_{s}\left(k^{-1} x\right) d k=\int_{K / U} \int_{U} \Delta_{s}\left(u^{-1} k^{-1} x\right) d u d k_{K / U} \\
& =\int_{K / U} \int_{U} \Delta_{s}\left(u k^{-1} x\right) d u d k_{K / U} \\
& =(\operatorname{det} x)^{s_{r}} \int_{I_{1}} \Phi_{s-s_{r} \cdot 1^{r}}(P(e-c)(x)) d \nu(c) .
\end{aligned}
$$

We need to justify the last equality. Let $c=k e_{r} \in I_{1}$ and let $\operatorname{det}_{j}$ and $V_{j}$ be as in Definition 3.1. Now, $\Delta_{r}\left(k u k^{-1} x\right)=\operatorname{det}\left(k u k^{-1} x\right)=\operatorname{det}(x)$ and, if $1 \leq j \leq r-1$,

$$
\begin{aligned}
\Delta_{j}\left(u k^{-1}\right) & =\operatorname{det}_{j}\left(\operatorname{proj}_{V_{j}}\left(u k^{-1} x\right)\right)=\operatorname{det}_{j}\left(\operatorname{proj}_{V_{j}}\left(u k^{-1} P(e-c)(x)\right)\right) \\
& =\operatorname{det}\left(\sum_{i=j+1}^{r} e_{i}+\operatorname{proj}_{V_{j}}\left(u k^{-1} P(e-c)(x)\right)\right) \\
& =\operatorname{det}\left(k^{-1}\left(\sum_{i=j+1}^{r} k e_{i}+\operatorname{proj}_{V_{j}^{\prime}}\left(k u k^{-1} P(e-c)(x)\right)\right)\right) \\
& =\operatorname{det}_{j}^{\prime}\left(\operatorname{proj}_{V_{j}^{\prime}}(v P(e-c)(x))\right),
\end{aligned}
$$


where $\operatorname{det}_{j}^{\prime}$ is the determinant relative to the Jordan algebra

$$
V_{j}^{\prime}=V\left(k e_{1}+\cdots+k e_{j}, 1\right)
$$

and $v=k u k^{-1} \in \tilde{U}=\{k \in K: k c=c\}=\bigcup_{i=1}^{N}(\operatorname{Aut}(V(c, 0)))_{0} k_{i}$ where $k_{i} \in \tilde{U}$. The latter is a disjoint union where $N$ does not depend on $c \in I_{1}$.

We need to verify that $P(e-c)(x) \in \Omega(V(c, 0))$. Suppose $x=\alpha c+x_{1 / 2}+x_{0}$ with $x_{i} \in V(c, i)$ (the Peirce decomposition with respect to $c$ ). It suffices to show that $L\left(x_{0}\right)$ is positive definite on $V(c, 0)$. If $y \neq 0 \in V(c, 0)$ then $\left(L\left(x_{0}\right) y \mid y\right)=$ $\left(x_{0} y \mid y\right)=\left(\left(x-x_{1 / 2}-\alpha c\right) y \mid y\right)=(x y \mid y)=(L(x) y \mid y)>0$.

\section{INTEGRATING OVER THE PRIMITIVE IDEMPOTENTS}

The main goal of this section is to prove:

Theorem 4.1. Let $\left\{e_{1}, \ldots, e_{r}\right\}$ be a complete orthogonal system of primitive idempotents. Given the Peirce decomposition $\sum_{i=1}^{r} \beta_{i} e_{i}+\sum_{i<j} c_{i j}$ of $c \in I_{1}$ with respect to that system, write $\beta_{i}(c)=\beta_{i}$. Then

$$
\begin{array}{rl}
\int_{I_{1}} & f\left(\beta_{1}(c), \ldots, \beta_{r}(c)\right) d \nu(c) \\
& =\frac{\Gamma(r m / 2)}{(\Gamma(m / 2))^{r}} \int_{\sigma} f\left(\beta_{1}, \ldots, \beta_{r}\right)\left(\prod_{i=1}^{r} \beta_{i}^{(m / 2)-1}\right) d \beta_{1} \cdots d \beta_{r-1},
\end{array}
$$

where $\sigma=\left\{\left(\beta_{1}, \ldots, \beta_{r}\right): \sum_{i=1}^{r} \beta_{i}=1\right.$ and $\beta_{i} \geq 0$ for each $\left.i\right\}$.

We will use induction on $r$, the rank of the Jordan algebra. An essential ingredient for this induction is the following result.

Lemma 4.2. Let $c \in I_{1}$. Let $\mathbf{S}^{m(r-1)-1}=\{y \in V(c, 1 / 2):\|y\|=1\}$, let be $d \nu(y)$ be the unique measure on $\mathbf{S}^{m(r-1)-1}$ which is invariant under the group of linear transformations of $V(c, 1 / 2)$ preserving $\|\cdot\|$ and such that $\int_{\mathbf{S}^{m(r-1)-1}} d \nu(y)=$ 1. Then if $I_{1}(V(c, 0))$ is the space of primitive idempotents of the Jordan algebra $V(c, 0)$, we have

$$
\int_{I_{1}(V(c, 0))} f(d) d \nu(d)=\int_{\mathbf{S}^{m(r-1)-1}} f\left(2 y^{2}-c\right) d \nu(y)
$$

$\left(d \nu(d)\right.$ is the usual measure on $I_{1}(V(c, 0))$ seen as a symmetric space).

Proof. From standard results and from the proof of [7, Theorem 2.2], it is clear that the map which sends $y \in \mathbf{S}^{m(r-1)-1}$ to $2 y^{2}-c \in I_{1}(V(c, 0))$ is surjective. It then suffices to realize that the measure on $I_{1}(V(c, 0))$ defined by $\mu(f)=$ $\int_{\mathbf{S}^{m(r-1)-1}} f\left(2 y^{2}-c\right) d \nu(y)$ is invariant under $\{k \in K: k c=c\}$. The connected component of that group which contains the identity can be identified naturally with the connected component of the group of automorphisms of $V(c, 0)$ which contains the identity.

4.1. An integral formula for the integration over the primitive idempotents. Let $\mathfrak{k}$ be the Lie algebra of $K$. Fix $c \in I_{1}$, let $U$ be the subgroup of $K$ that leaves $c$ unchanged and let $\mathfrak{u}$ be its Lie algebra. We have the following decomposition: $\mathfrak{k}=\mathfrak{u}+\mathfrak{p}_{*}$, where $\mathfrak{p}_{*}=\{4[L(x), L(c)]: x \in V(c, 1 / 2)\}$. If $\mathfrak{p}_{*}=i \mathfrak{p}$, then $\mathfrak{g}_{0}=\mathfrak{u}+\mathfrak{p}$ is the Cartan decomposition of the dual Lie algebra $\mathfrak{g}_{0}$ of $\mathfrak{k}$ (see [6] for details). In that context, the following computations are useful. 
For $x \in V(c, 1 / 2)$, we have according to [7]

$$
\begin{aligned}
e^{4[L(x), L(c)]} c= & \cos ^{2}(\sqrt{2}\|x\| / 2) c+\frac{1}{2} \frac{\sin \sqrt{2}\|x\|}{\sqrt{2}\|x\|} x \\
& +\sin ^{2}(\sqrt{2}\|x\| / 2) \frac{x^{2}-\left(\|x\|^{2} / 2\right) c}{\|x\|^{2} / 2}
\end{aligned}
$$

Let $x, y \in V(c, 1 / 2)$ and define $T_{x}: V(c, 1 / 2) \rightarrow V(c, 1 / 2)$ by

$$
T_{x}=4\left(L(x)^{2}-L\left(x^{2}\right)\right)
$$

Then

$$
\begin{aligned}
{[4[L(x), L(c)],[4[L(x), L(c)], 4[L(y), L(c)]]] } & =-16[[L(x), L(c)],[L(x), L(y)]] \\
& =4\left[L\left(T_{x}(y)\right), L(c)\right] .
\end{aligned}
$$

The first equality is a consequence of $[7,(3.5)]$. To check the second equality, it suffices to apply both sides to $y_{i} \in V(c, i)$ for $i=0,1 / 2$ and 1 . Now, there exists $C \neq 0$ such that

$$
\int_{I_{1}} f(d) d \nu(d)=C \int_{\|x\|<\pi / \sqrt{2}} f\left(e^{4[L(x), L(c)]} c\right) \operatorname{det}\left(\sum_{n=0}^{\infty} \frac{T_{x}^{n}}{(2 n+1) !}\right) d x
$$

(see for instance [5], where the volume element is derived in the noncompact case).

Lemma 4.3. Let $c$ be a primitive idempotent and let $x \in V(c, 1 / 2)$ be nonzero. The eigenvalues of the map $T_{x}$ given above are 0 (with multiplicity 1$),-2\|x\|^{2}$ (with multiplicity $m-1$ ) and $-\|x\|^{2} / 2$ (with multiplicity $m(r-2)$ ).

Proof. There exists $\left\{e_{1}, \ldots, e_{r}\right\}$, a complete system of orthogonal primitive idempotents, such that $x=\sum_{i=1}^{r} \lambda_{i} e_{i}$. Since we have $\sum_{i=1}^{r} \lambda_{i}=0$ we can, without loss of generality, assume that $\lambda_{1}$ and $\lambda_{2}$ are nonzero. Now, we can write $c=\sum_{i=1}^{r} \beta_{i} e_{i}+\sum_{i<j} c_{i j}$, where $\sum_{i=1}^{r} \beta_{i}=1$ with $\beta_{i} \geq 0$ for each $i$ and $c_{i j} \in V\left(e_{i}, 1 / 2\right) \cap V\left(e_{j}, 1 / 2\right)$ (Lemma 5.1). From $c x=x / 2$, we see easily that $\beta_{i} \lambda_{i}=\lambda_{i} / 2$ for each $i$. This in turn implies that $\beta_{1}=\beta_{2}=1 / 2$ and that $\beta_{i}=0$ for $i>2$. It follows then that $\lambda_{1}=-\lambda_{2}= \pm\|x\| / \sqrt{2}$ and that $\lambda_{i}=0$ for $i>2$. Furthermore, we get that $c_{i j}=0$ unless $i=1$ and $j=2$, and that $\left\|c_{12}\right\|^{2}=1 / 2$ (again, Lemma 5.1). Similar considerations show that if $y \in V(c, 1 / 2)$, then $y=\alpha x+y_{12}+\sum_{j>2} y_{1 j}+\sum_{j>2} y_{2 j}$, where $\alpha$ is a scalar, $\lambda\left(y_{12} c_{12}\right)=0$ and $y_{i j} \in V\left(e_{i}, 1 / 2\right) \cap V\left(e_{j}, 1 / 2\right)$. The rest follows easily from that.

Corollary 4.4. $\operatorname{det}\left(\sum_{n=0}^{\infty} \frac{T_{x}^{n}}{(2 n+1) !}\right)=\left(\frac{\sin (\sqrt{2}\|x\|)}{\sqrt{2}\|x\|}\right)^{m-1}\left(\frac{\sin (\sqrt{2}\|x\| / 2)}{\sqrt{2}\|x\| / 2}\right)^{m(r-2)}$.

Proof. A direct consequence of Lemma 4.3. 


\subsection{Proof of Theorem 4.1.}

Proof. We incorporate the results of Lemma 4.2 and Corollary 4.4 with equations (2) and (3), taking $c=e_{1}$. Let $u=\sqrt{2}\|x\|$ and $y=x /\|x\|=\sqrt{2} x / u$.

(4)

$$
\begin{aligned}
\int_{I_{1}} f(d) d \nu(d) & \\
= & C \int_{\mathbf{S}^{m(r-1)-1}} \int_{0}^{\pi} f\left(\cos ^{2}(u / 2) e_{1}+\frac{1}{2^{3 / 2}}(\sin u) y+\sin ^{2}(u / 2)\left(2 y^{2}-e_{1}\right)\right) \\
& \cdot\left(\frac{2 \sin (u / 2) \cos (u / 2)}{u}\right)^{m-1}\left(\frac{\sin (u / 2)}{u / 2}\right)^{m(r-2)} u^{m(r-1)-1} d u d \nu(y) \\
= & \frac{\Gamma(m r / 2)}{(\Gamma(m / 2))^{r}} \int_{\mathbf{S}^{m(r-1)-1}} \int_{0}^{1} f\left(\beta_{1} e_{1}+\frac{1}{2^{3 / 2}}\left(\sin u\left(\beta_{1}\right)\right) y+\left(1-\beta_{1}\right)\left(2 y^{2}-e_{1}\right)\right) \\
& \cdot \beta_{1}^{(m / 2)-1}\left(1-\beta_{1}\right)^{m(r-1) / 2-1} d \beta_{1} d \nu(y) \quad \text { with } \beta_{1}=\cos ^{2}(u / 2) .
\end{aligned}
$$

We chose $C$ so that $\int_{I_{1}} d \nu(d)=1$ (since we assume that $\int_{\mathbf{S}^{m(r-1)-1}} d \nu(y)=1$ ). To complete the proof, we use induction on $r \geq 2$. Suppose

$$
f(d)=g\left(\beta_{1}(d), \ldots, \beta_{r}(d)\right) .
$$

If $r=2$, (4) becomes

$$
\int_{I_{1}} f(d) d \nu(d)=\frac{\Gamma(m)}{(\Gamma(m / 2))^{2}} \int_{0}^{1} g\left(\beta_{1}, \beta_{2}\right) \beta_{1}^{(m / 2)-1} \beta_{2}^{(m / 2)-1} d \beta_{1}
$$

where $\beta_{2}=1-\beta_{1}$. Suppose the result is true for $r-1$, where $r \geq 3$. It is clear from (4) and Lemma 4.2 that

$$
\begin{aligned}
& \int_{I_{1}} f(d) d \nu(d)=\frac{\Gamma(m r / 2)}{(\Gamma(m / 2))^{r}} \int_{0}^{1} \int_{\sigma^{\prime}} g\left(\beta_{1},\left(1-\beta_{1}\right)\left(\eta_{2}, \ldots, \eta_{r}\right)\right) \\
& \cdot \beta_{1}^{(m / 2)-1}\left(1-\beta_{1}\right)^{m(r-1) / 2-1} \eta_{2}^{(m / 2)-1} \ldots \eta_{r}^{(m / 2)-1} d \eta_{2} \ldots d \eta_{r-1} d \beta_{1},
\end{aligned}
$$

where $\sigma^{\prime}=\left\{\left(\eta_{2}, \ldots, \eta_{r}\right): \eta_{i} \geq 0, \sum_{i=2}^{r} \eta_{i}=1\right\}$. The result follows easily from there.

\section{The RECURSIVE FORMULA}

Lemma 5.1. Let $\left\{e_{1}, \ldots, e_{r}\right\}$ be a complete system of orthogonal primitive idempotents. Let $\sum_{i=1}^{r} \beta_{i} e_{i}+\sum_{i<j} c_{i j}$ be the Peirce decomposition of $c \in V$ with respect to that system. Then $c$ is a primitive idempotent if and only if

$$
\begin{aligned}
\sum_{i=1}^{r} \beta_{i} & =1 \text { and } \beta_{i} \geq 0 \text { for all } i, \\
\left\|c_{i j}\right\|^{2} & =2 \beta_{i} \beta_{j}(i \neq j), \\
c_{i s} c_{j s} & =(1 / 2) \beta_{s} c_{i j}(i, j \text { and } s \text { distinct }) .
\end{aligned}
$$

For convenience, $c_{i j}$ is interpreted here as $c_{j i}$ if $j<i$.

Proof. In the computations that follow, [2, Propositions IV.1.4 and IV.2.4] are particularly useful. Suppose that $c \in I_{1}$. From $c=c^{2}$, one finds also that $\beta_{p}=$ $\beta_{p}^{2}+\frac{1}{2} \sum_{j \neq p}\left\|c_{p j}\right\|^{2}$ for all $p$. From this, and from $\operatorname{tr} c=1$, one sees that the first 
condition is verified. It is also clear that $\beta_{p}=0$ if and only if $c_{p j}=0$ for all $j \neq p$. For any given $s$, we have $P(c)\left(e_{s}\right)=\alpha_{s} c$ for some scalar $\alpha_{s}$. If we expand the left hand side of that equation and use the above, we find that $\beta_{s}^{2}=\alpha_{s} \beta_{s}$, $c_{i s} c_{j s}=(1 / 2) \alpha_{s} c_{i j}$ and $\left\|c_{i s}\right\|^{2}=2 \alpha_{s} \beta_{i}$ (i,j and $s$ distinct). If $\beta_{s} \neq 0$, we have that $\alpha_{s}=\beta_{s}$, which gives us the second and third conditions for these indices. If $\beta_{s}=0$, then these conditions are true anyway since this implies that $c_{i s}=0$ for each $s \neq i$. It is easily verified that the conditions are sufficient.

Lemma 5.2. Let $x=\lambda_{1} e_{1}+\cdots+\lambda_{r} e_{r}$, where $\left\{e_{1}, \ldots, e_{r}\right\}$ is a complete system of orthogonal primitive idempotents and $\lambda_{1}>\lambda_{2}>\cdots>\lambda_{r}>0$. If $\sum_{i=1}^{r} \beta_{i} e_{i}+$ $\sum_{i<j} c_{i j}$ is the Peirce decomposition of $c \in I_{1}$ with respect to that system and $P\left(e_{i}\right)(c) \neq 0$ for $1 \leq i \leq r$, then $P(e-c)(x)=\sum_{i=1}^{r} \mu_{i} w_{i}$, where $\left\{w_{1}, \ldots, w_{r-1}\right\}$ is a complete system of primitive idempotents of $V(c, 0)$ and

$$
\begin{gathered}
\lambda_{1}>\mu_{1}>\lambda_{2}>\mu_{2}>\cdots>\lambda_{n-1}>\mu_{n-1}>\lambda_{n} \\
w_{k}=\left[\sum_{i=1}^{r} \frac{\beta_{i}}{\left(\lambda_{i}-\mu_{k}\right)^{2}} e_{i}+\sum_{i<j} \frac{c_{i j}}{\left(\lambda_{i}-\mu_{k}\right)\left(\lambda_{j}-\mu_{k}\right)}\right] /\left[\sum_{i=1}^{r} \frac{\beta_{i}}{\left(\lambda_{i}-\mu_{k}\right)^{2}}\right], \\
\beta_{p}=\frac{\prod_{i=1}^{r-1}\left(\mu_{i}-\lambda_{p}\right)}{\prod_{i \neq p}\left(\lambda_{i}-\lambda_{p}\right)} .
\end{gathered}
$$

Conversely, given $\mu_{1}, \ldots, \mu_{n-1}$ satisfying (5), there exists a primitive idempotent c for which the conditions of the lemma are satisfied.

Proof. In what follows, we will use Lemma 5.1 repeatedly. Suppose

$$
w=\sum_{i=1}^{r} \eta_{i} e_{i}+\sum_{i<j} w_{i j}
$$

is the Peirce decomposition of a primitive idempotent appearing in the spectral decomposition of $x_{0}=P(e-c)(x)$ and that $w$ corresponds to the eigenvalue $\mu$. We then have $x_{0} w=\mu w$.

Using the Peirce decomposition of $x$ with respect to $c$ and the relation $x_{0} w=\mu w$, we have $x w-\mu w \in V(c, 1 / 2)$. Using this fact and the Peirce decomposition of $x$ with respect to $w$, we have $x w-\mu w \in V(w, 1 / 2)$. Hence, $x w-\mu w \in$ $V(c, 1 / 2) \cap V(w, 1 / 2)$, which implies that

$$
(x w-\mu w)^{2}=\frac{1}{2}\|x w-\mu w\|^{2}(w+c) .
$$

Since

$$
x w-\mu w=\sum_{i=1}^{r}\left(\lambda_{i}-\mu\right) \eta_{i} e_{i}+\sum_{i<j}\left(\frac{\lambda_{i}+\lambda_{j}}{2}-\mu\right) w_{i j},
$$


it follows that

$$
\begin{aligned}
\frac{1}{2}\|x w-\mu w\|^{2} & =\frac{1}{2}\left[\sum_{i=1}^{r}\left(\lambda_{i}-\mu\right)^{2} \eta_{i}^{2}+\sum_{i<j}\left(\frac{\lambda_{i}+\lambda_{j}}{2}-\mu\right)^{2}\left\|w_{i j}\right\|^{2}\right] \\
& =\frac{1}{2}\left[\sum_{i=1}^{r}\left(\lambda_{i}-\mu\right)^{2} \eta_{i}^{2}+2 \sum_{i<j}\left(\frac{\lambda_{i}+\lambda_{j}}{2}-\mu\right)^{2} \eta_{i} \eta_{j}\right] \\
& =\frac{1}{2}\left[\sum_{i=1}^{r} \sum_{j=1}^{r}\left(\frac{\lambda_{i}+\lambda_{j}}{2}-\mu\right)^{2} \eta_{i} \eta_{j}\right] \\
& =\frac{1}{4}\left(\sum_{i=1}^{r}\left(\lambda_{i}-\mu\right)^{2} \eta_{i}+\left[\sum_{i=1}^{r}\left(\lambda_{i}-\mu\right) \eta_{i}\right]^{2}\right) \\
& =\frac{1}{4}\left(A+B^{2}\right) .
\end{aligned}
$$

Since $\operatorname{tr} z=0$ whenever $z \in V(d, 1 / 2)$, where $d$ is an idempotent, we find that

$$
B=\sum_{i=1}^{r}\left(\lambda_{i}-\mu\right) \eta_{i}=\operatorname{tr}(x w-\mu w)=0 .
$$

If we consider the coefficient of $e_{i}$ in the Peirce decomposition of $(x w-\mu w)^{2}=$ $\frac{A}{4}(w+c)$, we obtain

$$
\begin{array}{r}
\left(\lambda_{i}-\mu\right)^{2} \eta_{i}^{2}+\frac{1}{2} \sum_{p<i}\left(\frac{\lambda_{i}+\lambda_{p}}{2}-\mu\right)^{2}\left\|w_{p i}\right\|^{2}+\frac{1}{2} \sum_{p>i}\left(\frac{\lambda_{i}+\lambda_{p}}{2}-\mu\right)^{2}\left\|w_{i p}\right\|^{2} \\
=\frac{A}{4}\left(\eta_{i}+\beta_{i}\right) .
\end{array}
$$

This is the same as

$$
\begin{aligned}
\left(\lambda_{i}-\mu\right)^{2} \eta_{i}^{2}+\eta_{i} \sum_{p<i}\left(\frac{\lambda_{i}+\lambda_{p}}{2}-\mu\right)^{2} \eta_{p}+\eta_{i} \sum_{p>i}\left(\frac{\lambda_{i}+\lambda_{p}}{2}-\mu\right)^{2} \eta_{p} & \\
& =\frac{A}{4}\left(\eta_{i}+\beta_{i}\right) .
\end{aligned}
$$

This is also the same as $\eta_{i} \sum_{p=1}^{r}\left(\left(\lambda_{i}-\mu\right)+\left(\lambda_{p}-\mu\right)\right)^{2} \eta_{p}=A\left(\eta_{i}+\beta_{i}\right)$ or, using (7), $\eta_{i}\left(\lambda_{i}-\mu\right)^{2}+\eta_{i} \sum_{p=1}^{r}\left(\lambda_{p}-\mu\right)^{2} \eta_{p}=A\left(\eta_{i}+\beta_{i}\right)$; if one adds over $i, 1 \leq i \leq r$, one finds that $A=\sum_{p=1}^{r}\left(\lambda_{p}-\mu\right)^{2} \eta_{p}$. This, with the previous equation, implies that $\left(\lambda_{i}-\mu\right)^{2} \eta_{i}=A \beta_{i}$.

$A=\sum_{p=1}^{r}\left(\lambda_{p}-\mu\right)^{2} \eta_{p}$ cannot be 0 . Suppose it is; since by Lemma 5.1 , the $\eta_{p}$ cannot all be 0 , it means that $\mu=\lambda_{p}$ for some $p$ and that $\eta_{i}=0$ for $i \neq p$. We must then have $w=e_{p}$ (recall that $\eta_{i}=0$ implies $w_{p i}=0=w_{p j}$ for all $i$ and $j$ not equal to $p$ ). But $c w=0$ implies $\beta_{p}=0$, which is not true since we assumed $P\left(e_{p}\right)(c) \neq 0$ : contradiction. The equation $\left(\lambda_{i}-\mu\right)^{2} \eta_{i}=A \beta_{i}$ in turn implies that $\mu \neq \lambda_{i}$ for all $i$, since the $\beta_{i}$ are nonzero. Hence, for each $i, \eta_{i}=A \frac{\beta_{i}}{\left(\lambda_{i}-\mu\right)^{2}}$. Summing both sides over $1 \leq i \leq r$, we get $1=A \sum_{i=1}^{r} \frac{\beta_{i}}{\left(\lambda_{i}-\mu\right)^{2}}$. The projection on $V_{i j}$ of the equality 


$$
\begin{aligned}
& \frac{A}{4}(w+c)=(x w-\mu w)^{2} \text { gives } \\
& \qquad \frac{A}{4}\left(w_{i j}+c_{i j}\right)=\sum_{s=1}^{r} \eta_{s}\left(\frac{\lambda_{i}+\lambda_{s}}{2}-\mu\right)\left(\frac{\lambda_{s}+\lambda_{j}}{2}-\mu\right) w_{i j}=D_{i j} w_{i j}
\end{aligned}
$$

and

$$
\begin{aligned}
4 D_{i j}= & \sum_{s=1}^{r} \eta_{s}\left(\left(\lambda_{i}-\mu\right)+\left(\lambda_{s}-\mu\right)\right)\left(\left(\lambda_{j}-\mu\right)+\left(\lambda_{s}-\mu\right)\right) \\
= & \left(\lambda_{i}-\mu\right)\left(\lambda_{j}-\mu\right) \sum_{s=1}^{r} \eta_{s}+\left(\lambda_{i}+\lambda_{j}-2 \mu\right) \sum_{s=1}^{r} \eta_{s}\left(\lambda_{s}-\mu\right) \\
& +\sum_{s=1}^{r} \eta_{s}\left(\lambda_{s}-\mu\right)^{2} \\
= & \left(\lambda_{i}-\mu\right)\left(\lambda_{j}-\mu\right)+B+A=\left(\lambda_{i}-\mu\right)\left(\lambda_{j}-\mu\right)+A .
\end{aligned}
$$

If we put everything together, we find that $w_{i j}=A \frac{c_{i j}}{\left(\lambda_{i}-\mu\right)\left(\lambda_{j}-\mu\right)}$. Since this is true for every eigenvalue $\mu$ of $P(e-c)(x)$, this implies (6).

Let $\mu_{1}>\cdots>\mu_{r-1}$ be the eigenvalues of $P(e-c)(x)$. From (7) and from the expression for $\eta_{i}$, we have $\sum_{s=1}^{r} \frac{\beta_{s}}{\lambda_{s}-\mu_{i}}=0$. If $i<j$, then

$$
0=\sum_{s=1}^{r} \frac{\beta_{s}}{\lambda_{s}-\mu_{i}}-\sum_{s=1}^{r} \frac{\beta_{s}}{\lambda_{s}-\mu_{j}}=\left(\mu_{i}-\mu_{j}\right) \sum_{s=1}^{r} \frac{\beta_{s}}{\left(\lambda_{s}-\mu_{i}\right)\left(\lambda_{s}-\mu_{j}\right)},
$$

which means that we cannot have $i<j$ and $\lambda_{p}>\mu_{i}>\mu_{j}>\lambda_{p+1}$. We then have $\lambda_{1}>\mu_{1}>\lambda_{2}>\mu_{2}>\cdots>\lambda_{n-1}>\mu_{r-1}>\lambda_{r}$.

Let $\nu_{i}=\frac{1}{\mu_{i}}$ for $1 \leq i \leq r-1$ and $\nu_{n}=0$. We have

$$
\sum_{k=1}^{r} \frac{\beta_{s}}{1-\nu_{i} \lambda_{s}}= \begin{cases}0 & \text { if } 1 \leq i \leq r-1 \\ 1 & \text { if } i=n\end{cases}
$$

Using the formula

$$
\operatorname{det}\left[\frac{1}{1-\alpha_{i} \beta_{k}}\right]_{1 \leq i, k \leq r}=\frac{\prod_{i>k}\left(\alpha_{i}-\alpha_{k}\right) \prod_{i>k}\left(\beta_{i}-\beta_{k}\right)}{\prod_{i=1}^{r} \prod_{k=1}^{r}\left(1-\alpha_{i} \beta_{k}\right)}
$$

of [16, page 202] and Cramer's rule, we find that

$$
\beta_{p}=\frac{\prod_{i=1}^{r-1}\left(\mu_{i}-\lambda_{p}\right)}{\prod_{i \neq p}\left(\lambda_{i}-\lambda_{p}\right)}
$$

The converse is easily verified using Lemma 5.1.

Theorem 5.3. In the notation of Definition 3.1 and Lemma 3.2, if $r=1$ and $\lambda=\left(\lambda_{1}\right)$ with $\lambda_{1}>0$, then $\Phi_{s}(\lambda)=\lambda_{1}^{s_{1}}$. If $r>1$ and $\lambda=\left(\lambda_{1}, \ldots, \lambda_{r}\right)$ with 


$$
\begin{aligned}
\lambda_{1}>\lambda_{2}>\cdots>\lambda_{r}> & 0, \text { then } \\
\Phi_{s}\left(\lambda_{1}, \ldots, \lambda_{r}\right)= & \frac{\Gamma(m r / 2)}{(\Gamma(m / 2))^{r}} \prod_{i=1}^{r} \lambda_{i}^{s_{r}} \\
& \cdot \int_{\sigma} \Phi_{s-s_{r} \cdot 1^{r}}\left(\sum_{j=1}^{r} \beta_{j} e_{i}^{(j)}(\lambda)\right)_{1 \leq i \leq r-1}^{r}\left(\beta_{1} \cdots \beta_{r}\right)^{(m / 2)-1} d \beta \\
= & \frac{\Gamma(m r / 2)}{(\Gamma(m / 2))^{r}} \prod_{i<j}\left(\lambda_{i}-\lambda_{j}\right)^{1-m} \prod_{i=1}^{r} \lambda_{i}^{s_{r}} \\
& \cdot \int_{\lambda_{r}}^{\lambda_{r-1}} \ldots \int_{\lambda_{2}}^{\lambda_{1}} \Phi_{s-s_{r} \cdot 1^{r}}\left(\mu_{1}, \ldots, \mu_{r-1}\right) \\
& \cdot \prod_{i=1}^{r-1} \prod_{j=1}^{r}\left|\mu_{i}-\lambda_{j}\right|^{m / 2-1} \prod_{i<p}\left(\mu_{i}-\mu_{p}\right) d \mu_{1} \ldots d \mu_{r-1}
\end{aligned}
$$

where, in the first equality, $\sigma=\left\{\left(\beta_{1}, \ldots, \beta_{r}\right): \sum_{i=1}^{r} \beta_{i}=1\right.$ and $\beta_{i} \geq 0$ for each $\left.i\right\}$, we express $\Phi_{s-s_{r} \cdot 1^{r}}\left(\mu_{1}, \ldots, \mu_{r-1}\right)$ as a function of the elementary symmetric polynomials $e_{q}(\mu), 1 \leq q \leq r-1$, and write $e_{q}^{(j)}(\lambda)=e_{q}\left(\lambda_{1}, \ldots, \lambda_{j-1}, \lambda_{j+1}, \ldots, \lambda_{r}\right)$.

Proof. If we refer to Lemma 3.2 , we need to compute the measure $d \nu(c)$ there in terms of the eigenvalues $\mu_{i}$ of $P(e-c)(x)$. Most of that work is done in Lemma 5.2 (the restrictions on $c$ there only concern a set of measure 0 ). Using straightforward calculus,

$$
\frac{D\left(\beta_{i}\right)}{D\left(\mu_{j}\right)}=\frac{\prod_{i<p}\left(\mu_{i}-\mu_{p}\right)}{\prod_{i<p}\left(\lambda_{i}-\lambda_{p}\right)} .
$$

Note too that $\beta_{p}=\prod_{i=1}^{r-1}\left(\mu_{i}-\lambda_{p}\right) / \prod_{i \neq p}\left(\lambda_{i}-\lambda_{p}\right)$ for $1 \leq p \leq r$ is equivalent to $\sum_{i=1}^{r} \beta_{p}=1$ and $e_{i}(\mu)=\sum_{j=1}^{r} \beta_{j} e_{i}^{(j)}(\lambda)$ for $1 \leq i \leq r-1$. From that and from Theorem 4.1, the conclusion is straightforward.

The work leading to Theorem 5.3 is, in a way, a refinement of [12, Chapter 1].

Note that in the case $r=2$, we have

$$
\Phi_{s}\left(\lambda_{1}, \lambda_{2}\right)=\lambda_{1}^{s_{1}} \lambda_{2}^{s_{2}}{ }_{2} F_{1}\left(-\left(s_{1}-s_{2}\right), m / 2 ; m ;\left(\lambda_{1}-\lambda_{2}\right) / \lambda_{1}\right) .
$$

Definition 5.4. Let $m$ be any complex number with $\Re m>0$ ( $\Re=$ real part). We define the function $\Phi_{s}^{(m)}(\lambda)$ recursively as in Theorem 5.3 (replacing $\Phi_{s}(\lambda)$ by $\Phi_{s}^{(m)}(\lambda)$ to indicate the dependence on the parameter $m$ ).

Lemma 5.5. Let $D_{1}=\sum_{i=1}^{r} \lambda_{i} \frac{\partial}{\partial \lambda_{i}}$. Then the functions $\Phi_{s}^{(m)}(\lambda)$ are smooth, $\Phi_{s}^{(m)}(1, \ldots, 1)=1$ and $D_{1} \Phi_{s}^{(m)}(\lambda)=\left(s_{1}+\cdots+s_{r}\right) \Phi_{s}^{(m)}(\lambda)$.

Proof. Using the first equality of Theorem 5.3 and induction, the result follows easily (one finds that $\Phi_{s}^{(m)}$ is homogeneous of degree $s_{1}+\cdots+s_{r}$ ).

Theorem 5.6. Suppose that $\Re m>0$ and that $s$ is a partition of length at most $r$. If $\lambda=\left(\lambda_{1}, \ldots, \lambda_{r}\right)$ with $\lambda_{1}>\lambda_{2}>\cdots>\lambda_{r}>0$, then

$$
J_{s}^{(2 / m)}\left(\lambda_{1}, \ldots, \lambda_{r}\right)=(2 / m)^{|s|} \prod_{i=1}^{r}(m(r-i+1) / 2)_{s_{i}} \Phi_{s}^{(m)}\left(\lambda_{1}, \ldots, \lambda_{r}\right) .
$$


This is a generalization of [17, Proposition 2.4], which corresponds to the case $r=2$.

Proof. For $m=1,2,4$ and $8(r=3)$ this is a consequence of Theorem 5.3 and of the relation between the spherical functions of symmetric cones and Jack polynomials.

We need to check that the three conditions that characterize $J_{s}^{(2 / m)}(\lambda)$, as given on page 3571, are satisfied. The first equality of Theorem 5.3, and induction, guarantee that $\Phi_{s}^{(m)}(\lambda)$ is a symmetric polynomial of the form $\sum_{t \leq s} v_{s t}(\alpha) m_{t}(\lambda)$. The second condition is clear since $\Phi_{s}^{(m)}(1, \ldots, 1)=1$ by Lemma 5.5. It suffices then to show that $D_{2}(m) \Phi_{s}^{(m)}(\lambda)=\gamma(2 / m, r, s) \Phi_{s}^{(m)}(\lambda)$, where $D_{2}$ is given by (1).

Let $H(\lambda)=\left\{\mu=\left(\mu_{1}, \ldots, \mu_{r-1}\right): \lambda_{i}>\mu_{i}>\lambda_{i+1}, i=1, \ldots, n-1\right\}, d \nu(\mu)=$ $\prod_{i<j}\left(\mu_{i}-\mu_{j}\right)^{m} /\left(\mu_{1}^{2} \cdots \mu_{r-1}^{2}\right) d \mu$ and

$$
\begin{aligned}
S_{m}(\lambda, \mu)= & \frac{\Gamma(m r / 2)}{(\Gamma(m / 2))^{r}} \prod_{i<j}\left(\lambda_{i}-\lambda_{j}\right)^{1-m} \prod_{i<j}\left(\mu_{i}-\mu_{j}\right)^{1-m} \\
& \cdot \prod_{i=1}^{r-1} \prod_{j=1}^{r}\left|\mu_{i}-\lambda_{j}\right|^{(m / 2)-1} \prod_{i=1}^{r-1} \mu_{i}^{2} .
\end{aligned}
$$

Assuming for the moment that $\Re m$ is large enough, we can make use of the fact that $S_{m}(\lambda, \mu)$ and its derivatives of low order are 0 on the boundary of $H(\lambda)$. The differential operator $D_{2}(m)$ is as in $(1)$, and $D_{2}^{\prime}(m)$ is the corresponding operator in the $r-1$ variables $\mu_{i}$ (similarly for $D_{1}$ introduced in Lemma 5.5 and for $D_{1}^{\prime}$ ). The functions $\Phi_{s}^{(m)}$ being as above, we have

$$
\begin{aligned}
D_{2}(m) & \Phi_{s}^{(m)}(\lambda) \\
= & r s_{r}\left(s_{r}-1+m(r-1) / 2\right) \prod_{i=1}^{r} \lambda_{i}^{s_{r}} \int_{H(\lambda)} \Phi_{s-s_{r} \cdot 1^{r}}^{(m)}(\mu) S_{m}(\lambda, \mu) d \nu(\mu) \\
& +2 s_{r} \prod_{i=1}^{r} \lambda_{i}^{s_{r}} \int_{H(\lambda)} \Phi_{s-s_{r} \cdot 1^{r}}^{(m)}(\mu) D_{1} S_{m}(\lambda, \mu) d \nu(\mu) \\
& +\prod_{i=1}^{r} \lambda_{i}^{s_{r}} \int_{H(\lambda)} \Phi_{s-s_{r} \cdot 1^{r}}^{(m)}(\mu) D_{2}(m) S_{m}(\lambda, \mu) d \nu(\mu) \\
= & r s_{r}\left(s_{r}-1+m(r-1) / 2\right) \Phi_{s}^{(m)}(\lambda) \\
& +2 s_{r} \prod_{i=1}^{r} \lambda_{i}^{s_{r}} \\
& \cdot \int_{H(\lambda)} \Phi_{s-s_{r} \cdot 1^{r}}^{(m)}(\mu)\left(-D_{1}^{\prime}+\frac{(r-1)(2+(2-r) m)}{2}\right) S_{m}(\lambda, \mu) d \nu(\mu) \\
& +\prod_{i=1}^{r} \lambda_{i}^{s_{r}} \int_{H(\lambda)} \Phi_{s-s_{r} \cdot 1^{r}}^{(m)}(\mu) \\
& \cdot\left(D_{2}^{\prime}(m)-m D_{1}^{\prime}+m \frac{(r-1)(2+(2-r) m)}{2}\right) S_{m}(\lambda, \mu) d \nu(\mu) \\
= & r s_{r}\left(s_{r}-1+m(r-1) / 2\right) \Phi_{s}^{(m)}(\lambda) \\
& +\left(2 s_{r}+m\right) \prod_{i=1}^{r} \lambda_{i}^{s_{r}} \int_{H(\lambda)} D_{1}^{\prime} \Phi_{s-s_{r} \cdot 1^{r}}^{(m)}(\mu) S_{m}(\lambda, \mu) d \nu(\mu)
\end{aligned}
$$




$$
\begin{aligned}
& +\prod_{i=1}^{r} \lambda_{i}^{s_{r}} \int_{H(\lambda)} D_{2}^{\prime}(m) \Phi_{s-s_{r} \cdot 1^{r}}^{(m)}(\mu) S_{m}(\lambda, \mu) d \nu(\mu) \\
= & {\left[r s_{r}\left(s_{r}-1+m(r-1) / 2\right)\right.} \\
& \left.\quad+\left(2 s_{r}+m\right) \sum_{i=1}^{r-1}\left(s_{i}-s_{r}\right)+\gamma\left(m, r-1, s-s_{r} \cdot 1^{r}\right)\right] \Phi_{s-s_{r} \cdot 1^{r}}^{(m)}(\mu) \\
= & \gamma(m, r, s) \Phi_{s}^{(m)}(\lambda) .
\end{aligned}
$$

The first equality is obtained by applying $D_{2}(m)$ inside the integral (recall that $\Re m$ is "large enough"). The second equality is just a matter of computing the respective terms. The third equality is just an application of integration by parts $\left(D_{2}^{\prime}(m)\right.$ is symmetric with respect to the measure $d \nu(\mu)$ and the transpose of $-D_{1}^{\prime}+\frac{(r-1)(2+(2-r) m)}{2}$ with respect to that measure is $\left.D_{1}^{\prime}\right)$. The fourth equality is a consequence of the induction hypothesis and of Lemma 5.5.

Finally, using analytic continuation allows us to remove the restriction on $\Re m$.

Corollary 5.7. The functions $\Phi^{(m)}(\lambda)$ can be extended analytically to $m \in \mathbf{C}-$ $\{-2 p / q: q=2,3, \ldots, r$ and $p>0$ is an integer not a multiple of $q\}$.

Proof. Clear from the first equation of Theorem 5.3.

Definition 5.8. Let $\lambda=\left(\lambda_{1}, \ldots, \lambda_{r}\right)$. If $l(s) \leq r$, write

$$
J_{s}^{(2 / m)}(\lambda)=\sum_{t \leq s} d_{s t}(2 / m) e_{t^{\prime}}(\lambda)
$$

Define $\mathcal{S}_{s}^{(r)}=\left\{m \in \mathbf{C}^{*}: 2 / m\right.$ is a pole of one of the functions $\left.d_{s t}\right\}$.

Conjecture 5.9 (Macdonald). The coefficients $d_{s t}(\alpha)$ are polynomial functions of $\alpha$.

Since the coefficients $d_{s t}$ are rational functions, they are all polynomial functions if and only if $\mathcal{S}_{s}^{(r)}=\emptyset$ (since the coefficients $d_{s t}$ are analytic at 0$)$. The conjecture is also equivalent to $J_{s}^{(2 / m)}(\lambda)$ being an analytic function for $m \in \mathbf{C}^{*}$. Note also that $\mathcal{S}_{s}^{(r)} \subset \mathcal{S}_{s}^{(r+1)}$ for each $r$, since $J_{s}^{(2 / m)}\left(\lambda_{1}, \ldots, \lambda_{r}\right)=J_{s}^{(2 / m)}\left(\lambda_{1}, \ldots, \lambda_{r}, 0\right)$. Furthermore, $\mathcal{S}_{s}^{(r)} \subset \mathcal{S}_{s}^{(N)}$ for each $r \geq 1$, where $N=\operatorname{degree} J_{s}^{(2 / m)}$.

Lemma 5.10. We have $\mathcal{S}_{s}^{(1)}=\emptyset$ and, for $r \geq 2, \mathcal{S}_{s}^{(r)} \subset \mathcal{S}_{s-s_{r} \cdot 1^{r}}^{(r-1)} \cup\left\{-2 p / q: s_{i} \leq\right.$ $\left.p<s_{i-1}-s_{r}, i=2, \ldots, r, 1<q<r, q \nmid p\right\}$.

Proof. Let

$$
\begin{aligned}
K_{r, s}(m) & =J_{s}^{(2 / m)}\left(1^{r}\right) / J_{s-s_{r} \cdot 1^{r}}^{(2 / m)}\left(1^{r-1}\right) \\
& =(2 / m)^{r s_{r}}(m r / 2)_{s_{1}} \prod_{i=2}^{r} \frac{(m(r-i+1) / 2)_{s_{i}}}{(m(r-i+1) / 2)_{s_{i-1}-s_{r}}} .
\end{aligned}
$$


Using the first equality of Theorem 5.3 and the relation between $J_{s}^{(2 / m)}$ and $\Phi_{s}^{(m)}$, one has

$$
\begin{aligned}
J_{s}^{(2 / m)}(\lambda)= & K_{r, s}(m) \frac{\Gamma(m r / 2)}{(\Gamma(m / 2))^{r}} \prod_{i=1}^{r} \lambda_{i}^{s_{r}} \\
& \cdot \int_{\sigma} J_{s-s_{r} \cdot 1^{r}}^{(2 / m)}\left(\sum_{j=1}^{r} \beta_{j} e_{i}^{(j)}(\lambda)\right)_{1 \leq i \leq r-1}\left(\beta_{1} \cdots \beta_{r}\right)^{(m / 2)-1} d \beta \\
= & K_{r, s}(m) \frac{\Gamma(m r / 2)}{(\Gamma(m / 2))^{r}} \prod_{i=1}^{r} \lambda_{i}^{s_{r}} \sum_{|a| \leq s_{1}-s_{r}} \\
& \cdot \int_{\sigma} P_{a, s-s_{r} \cdot 1^{r}}^{(m)}\left(\lambda \beta_{1}^{a_{1}} \cdots \beta_{r}^{a_{r}}\left(\beta_{1} \cdots \beta_{r}\right)^{(m / 2)-1} d \beta\right. \\
= & \prod_{i=1}^{r} \lambda_{i}^{s_{r}} \sum_{|a| \leq s_{1}-s_{r}} P_{a, s-s_{r} \cdot 1^{r}}^{(m)} \frac{K_{r, s}(m) \prod_{i=1}^{r}(m / 2)_{a_{i}} .}{(m r / 2)_{|a|}}
\end{aligned}
$$

The poles of the functions $P_{a, s-s_{r} \cdot 1^{r}}^{(m)}(\lambda)$, if any, belong to $\mathcal{S}_{s-s_{r} \cdot 1^{r}}^{(r-1)}$. The term $(m r / 2)_{s_{1}}$ takes care of the denominator $(m r / 2)_{|a|}$. The additional poles, if any, will come from

$$
\prod_{i=2}^{r} \frac{(m(r-i+1) / 2)_{s_{i}}}{(m(r-i+1) / 2)_{s_{i-1}-s_{r}}} .
$$

Lemma 5.11. $4 / m \in \bigcup_{n \geq 1} \mathcal{S}_{\mathcal{s}^{\prime}}^{(n)}$ if and only if $m \in \bigcup_{n \geq 1} \mathcal{S}_{s}^{(n)}$.

Proof. For any partition $s$, we have $J_{s}^{(\alpha)}=\sum c_{s t}(\alpha) p_{t}$. If $4 / m_{0} \notin \bigcup_{n \geq 1} \mathcal{S}_{s^{\prime}}^{(n)}$, then $4 / m_{0} \notin \mathcal{S}_{s^{\prime}}^{\left(n_{0}\right)}$, where $n_{0}=\max \left\{l(t): c_{s^{\prime} t} \neq 0\right\}$. The set of polynomials $\left\{p_{t}\left(\eta_{1}, \ldots, \eta_{n_{0}}\right): c_{s^{\prime} t} \neq 0\right\}$ is then linearly independent; therefore the coefficients $c_{s^{\prime} t}$ must be analytic at $\alpha=2 /\left(4 / m_{0}\right)$. Now, [15, Corollary 3.5] implies that $J_{s}^{(2 / m)}=\sum(-2 / m)^{|s|-l(t)} c_{s^{\prime} t}(2 /(4 / m)) p_{t}$. We can conclude that $m_{0} \notin$ $\bigcup_{n \geq 1} \mathcal{S}_{s}^{(n)}$, since $J_{s}^{(2 / m)}$ is then analytic at $m=m_{0}$. The converse follows in the same way.

Lemma 5.12. Suppose $\lambda=\left(\lambda_{1}, \ldots, \lambda_{r}\right)$. Then

$$
J_{s}^{(2 / m)}(\lambda)=(2 / m)^{r s_{r}} \prod_{i=1}^{r}\left(m(r-i+1)+s_{i}-s_{r}\right)_{s_{r}} e_{r}^{s_{r}} J_{s-s_{r} \cdot 1^{r}}^{(2 / m)}(\lambda) .
$$

Proof. Clear from the first equality of Theorem 5.3 and the relation between $\Phi_{s}^{(2 / m)}$ and $J_{s}^{(2 / m)}$.

Corollary 5.13. $\mathcal{S}_{s}^{(r)} \subset \mathcal{S}_{s-s_{r} \cdot 1^{r}}^{(r)}$

Some partial results:

Proposition 5.14. Macdonald's conjecture is true for $r \leq 3$.

Proof. It is clear that $\mathcal{S}_{s}^{(r)}=\emptyset$ when $r \leq 2$.

Suppose $r=3$. Using Lemma 5.12, it clearly suffices to show that $\mathcal{S}_{s}^{(3)}=\emptyset$ for all $s$ with $s_{3}=0$. This means that $s^{\prime}=\left(2 \cdot 1^{s_{2}}, 1^{s_{1}-s_{2}}\right)$. Lemma 5.10 shows that 
for $n \geq 1, \mathcal{S}_{s^{\prime}}^{(n)} \subset\{-2 / q: 1<q<n\}$. Lemma 5.11 implies that if $\mathcal{S}_{s}^{(4)} \neq \emptyset$ then it can only contain even integers, which is absurd by Lemma 5.10.

\section{Conclusion}

The version of Theorem 5.3 given in our doctoral dissertation [12] is also used to "inspire" some of the results in [13] and in [14]. In both these papers, we need Fourier analysis on the symmetric space in order to study the heat kernel and derive appropriate bounds. In [13], modifying the domain of integration in Theorem 5.3 leads us to an expression for the inverse of the Abel transform (a result similar to finding the inverse of the Fourier transform). In [14], our goal is the same but our inspiration follows a much closer path to the present paper, although, in [14], $s$ is no longer a partition.

Theorem 5.6 provides a natural extension for the limited connection that already exists between the Jack polynomials and the spherical functions of symmetric cones. One can say that, up to a known constant multiple, the Jack polynomial parametrized by $\alpha$ and indexed by $s$ is the spherical function indexed by $s$ and associated to the root system $A_{r-1}$ for the multiplicity $2 / \alpha$. While the reasoning used in the proof of Proposition 5.14 does not work for $r \geq 4$, it can be hoped that a refinement would lead to a general proof of Macdonald's conjecture.

\section{ACKNOWLEDGMENT}

We are grateful to Professor Erhard Neher, with whom we had very useful discussions on symmetric cones and Jordan algebras. We also wish to thank the referee and the editor for their suggestions to improve the presentation of our results.

\section{REFERENCES}

1. H. Braun and M. Koecher, Jordan Algebren, Springer-Verlag, Berlin and Heidelberg, 1966. MR 34:4310

2. Jacques Faraut, Algèbres de Jordan et cônes symétriques, Ecole CIMPA - Université de Poitiers, 22 Août - 16 Septembre, 1988.

3. Jacques Faraut, Fonctions de Legendre sur les algèbres de Jordan, CWI Quarterly 5, no. 4, (1992), 309-320. MR 94e:33025

4. Kenneth I. Gross and D. Richards, Special functions of matrix argument. I: Algebraic induction, zonal polynomials, and hypergeometric functions, Transactions of the American Mathematical Society 301 (1987), 781-811. MR 88m:22018

5. Sigurdur Helgason, Differential operators on homogeneous spaces, Acta Math. 102 (1959), 239-299. MR 22:8457

6. Sigurdur Helgason, Differential Geometry, Lie Groups and Symmetric Spaces, Academic Press, New York, 1978. MR 80k:53081

7. Ulrich Hirzebruch, Über Jordan-Algebren und kompakte Riemannsche symmetrische Räume vom Rang 1, Math. Zeitschr. 90 (1965), 339-354. MR 32:6371

8. Carl S. Herz, Bessel functions of matrix arguments, Annals of Mathematics 61 (1955), 474-523. MR 16:1107e

9. I. G. Macdonald, Symmetric Functions and Hall Polynomials, Oxford University Press, 1979. MR 84g:05003

10. I. G. Macdonald, Commuting differential operators and zonal spherical functions, Lecture Notes in Mathematics, vol. 1271, Springer-Verlag, New York, 1987, pp. 189-200. MR 89e:43025

11. R. J. Muirhead, Aspects of Multivariate Statistical Theory, Wiley, New York, 1982. MR 84c: 62073

12. Patrice Sawyer, The heat equation on the symmetric space associated to $\mathbf{S L}(n, \mathbf{R})$, Thesis, McGill University, 1989. 
13. Patrice Sawyer, The heat equation on spaces of positive definite matrices, Canadian Journal of Mathematics 44 (1992), 624-651. MR 94c:58202

14. Patrice Sawyer, On an upper bound for the heat kernel on $\mathbf{S U}^{\star}(2 n) / \mathbf{S p}(n)$, Canadian Bulletin of Mathematics 37 (1994), 408-418. MR 95k:58156

15. Richard P. Stanley. Some combinatorial properties of Jack symmetric polynomials, Advances in Mathematics 77 (1989), 76-115. MR 90g:05020

16. Hermann Weyl, The Classical Groups, 3rd ed., Princeton University Press, Princeton, New Jersey, 1946. MR 1:42c (1st ed.)

17. ZhiMin Yan, Generalized hypergeometric functions and Laguerre polynomials in two variables, Contemporary Mathematics 138 (1992), 239-259. MR 94j:33019

Department of Mathematics and Computer Science, Laurentian University, Sudbury (Ontario) Canada P3E 2C6

E-mail address: sawyer@ramsey.cs.laurentian.ca 